\section{Journal of \\ Epilepsy and \\ Clinical \\ Neurophysiology}

J Epilepsy Clin Neurophysiol 2010;16(3):100-105

\title{
Mortalidade Atribuída à Epilepsia, às Suas Doenças Subjacentes ou às Condições Não Relacionadas a Ela: Uma Síntese*
}

\author{
Marleide da Mota Gomes
}

Instituto de Neurologia Deolindo Couto - Universidade Federal do Rio de Janeiro (UFRJ)

\begin{abstract}
RESUMO
Objetivos: Conhecer em relação à epilepsia: epidemiologia das causas diretas ou indiretas das mortes, inferências e críticas sobre dados de mortalidade a partir de declaração de óbitos. Refletir sobre as recomendações/alertas sobre o risco de morte prevenível a pacientes e familiares. Métodos: Revisão baseada em artigos publicados principalmente nos últimos três anos a partir de busca no Pubmed com os descritores epilepsy e mortality nos títulos. Acrescentadas mais referências sobre mortalidade/declaração de óbitos, ideação suicida, profilaxia de mortes preveníveis (inquérito e medidas). Resultados: Os estudos são os: com abrangência populacional a partir do Sistema de Informação em Saúde do Ministério da Saúde Brasileiro; sobre principais fatores de risco para morte prematura em pessoas com epilepsia; sobre óbitos relacionados à epilepsia (morte súbita em epilepsia - SUDEP, crise epiléptica ou estado de mal epiléptico, suicídio, acidente, cirurgia para epilepsia, estimulação nervo vago e gravidez); sobre óbitos não relacionados à epilepsia (principalmente, neoplasias, doenças cerebrovasculares, doença isquêmica coronariana e infecções respiratórias); sobre óbitos evitáveis e recomendações. Conclusões: Os pacientes com epilepsia têm a mortalidade aumentada em comparação à população geral. A questão da mortalidade aumentada ou não no pós-operatório de cirurgia para epilepsia ainda não está respondida. Os suicídios foram mais comuns particularmente nos com epilepsia refratária e/ou comorbidade psiquiátrica. SUDEP é um fenômeno epilepsia-relacionado, de maior ocorrência em pessoas jovens com epilepsia, multicausal; profilaxia das mortes evitáveis deveria ser feita pela individualização da informação a ser dada a cada paciente.
\end{abstract}

Unitermos: Epilepsia, mortalidade, síndrome de morte súbita em epilepsia.

\begin{abstract}
Mortality attributed to the epilepsy, its underlying diseases or the unrelated conditions to it: a synthesis

Objective: To know regarding the epilepsy: the epidemiology of the direct or indirect causes of the deaths; inferences and critics on data about mortality from death certificates and risk factors for the deaths. To reflect on the recommendations/warnings about the risk of preventable death in patients. Methods: Review based on articles published mainly in the last three years obtained from search in the Pubmed with the describers epilepsy and mortality in the headings. Added more references on mortality/death certificates, suicidal ideation and prophylaxis of preventable deaths (survey and measures). Results: The studies are those: based on the Mortality Database of the Brazilian Ministry of Health; about main factors of risk for premature death in people with epilepsy; about mortality related to the epilepsy (sudden unexpected death in epilepsy - SUDEP, seizure and "status epilepticus", suicide, accident, surgery for epilepsy, vagal nerve stimulation and pregnancy); about unrelated mortality from the epilepsy (mainly, neoplasias, cerebrovascular disease, coronary artery disease and respiratory infection); about preventable deaths prophylaxis. Conclusions: The mortality among people with epilepsy is higher than that of the general population. The question of the increased mortality, in the postoperative period of the surgery for epilepsy, is not yet answered. The suicide occurs more often, particularly in people with refractory epilepsy and/or psychiatric comorbidities. SUDEP is an epilepsy-related phenomenon, multicausal and with higher occurrence in epileptic young people. Preventable deaths prophylaxis should be carried out by the individualization, to each patient, of the provided information. Keywords: Epilepsy, mortality, sudden unexpected death in epilepsy.
\end{abstract}

\footnotetext{
* Trabalho apresentado parcialmente no XXXIII Congresso da Liga Brasileira de Epilepsia, 03-06 junho 2010 (Brasília/DF).

Received June 11, 2010; accepted July 16, 2010.
} 


\section{INTRODUÇÃO}

O óbito, o mais indesejado dos desfechos, é sabidamente de maior risco de acontecer nos pacientes com epilepsia do que nos indivíduos da população geral: duas a três vezes superior, mas, cinco vezes, nos com epilepsia focal farmacorresistente. ${ }^{1,2,3}$ As mortes podem ser atribuídas à epilepsia, às doenças responsáveis pela epilepsia ou às condições não relacionadas a ela. Este artigo apresenta inferências e críticas sobre dados de mortalidade no Brasil a partir de declaração de óbitos sobre epilepsia e fatores de risco para as mortes precoces (idade e gênero, tipo de crise epiléptica - CE e síndrome da epilepsia, déficits neurológicos congênitos e déficits de aprendizagem, duração e gravidade da epilepsia). São consideradas também a mortalidade relacionadas à $\mathrm{CE}$ ou epilepsia (SUDEP, EME, suicídio, acidente, cirurgia, estimulação nervo vago e gravidez) ou mortalidade não relacionada à epilepsia. É também abordada a questão da informação aos pacientes/familiares/cuidadores sobre mortalidade precoce e desnecessária relacionada ou não à SUDEP.

\section{METODOLOGIA}

A revisão foi baseada na busca de artigos no Pubmed com os descritores mortality e epilepsy nos títulos, nos últimos três anos (10.04.2010). Obtidos oito artigos. ${ }^{1-8}$ Acrescentadas mais uma referência sobre mortalidade/ declarações de óbitos, ${ }^{9}$ outra sobre ideação suicida ${ }^{10}$ e mais três sobre profilaxia das crises ${ }^{11,12}$ e inquérito sobre aconselhamento e recomendações relativas "às necessidades do paciente sobre conhecimento relativo à sua condição". ${ }^{13}$ A referência com a recomendação do National Institute for Health and Clinical Excellence (NICE) da Inglaterra e País de Gales sobre o esclarecimento relacionado à eventualidade de morte também foi apresentada. ${ }^{14}$

\section{Estudos com abrangência populacional: declaração de óbitos}

O uso de dados de mortalidade a partir de declaração de óbitos deve ser feita com cautela, mas pela sua abrangência, os dados podem ser úteis para avaliar tendências temporais e de acometimento de algumas populações sob risco. O Sistema de Informação em Saúde do Ministério da Saúde/ DATASUS pode ser muito útil para esse tipo de estudo. Isso se usados dados principalmente de capitais onde as causas mal definidas, sub-registro e subcoleta são das menores do Brasil e representativas das várias regiões. Em relação ao Brasil como um todo, usando dados de melhor qualidade a partir dos das capitais, observa-se maior mortalidade masculina em quase todas as faixas etárias, e também no(a)s anciãos(ãs). ${ }^{9}$ Embora a epilepsia não seja proporcionalmente importante entre todas as causas de óbitos, ela é mais representada dos 20 aos 49 anos de idade (frequência relativa por idade). ${ }^{9}$ Taxas ajustadas de mortalidade/100.000, no ano de 2007, são baixas em alguns capitais e mais altas em outras (Belém, 1,38; Teresina, 1,73). As taxas em capitais representativas das regiões brasileiras são: Recife, 0,25; Rio de Janeiro, 0,86; Porto Alegre, 1,05; Goiânia, 0,38. ${ }^{9}$ A mortalidade em domicílio está predominantemente na faixa etária de 20-49 anos, onde possivelmente ocorreriam algumas das mortes por SUDEP, enquanto a hospitalar, provavelmente relacionada a causas subjacentes cerebrais, nos idosos. ${ }^{9}$ Em relação às tendências temporais, no nosso estudo (apenas de capitais) referente ao período de 2003-2007, ${ }^{9}$ houve um incremento da mortalidade, com redução estimada pela mortalidade proporcional de 1979 a 2007, de 13\%. O estudo de Ferreira e Silva ${ }^{4}$ a partir da mesma fonte, mas com dados do Brasil todo, constatou uma redução de $20,35 \%$ na taxa de mortalidade de epilepsia no Brasil (1,13/100.000 em 1980 a 0,9/100.000 em 2003). No entanto, reconheceu também aumento em um determinado período $(0,83 / 100.000 \mathrm{em}$ 1994 a 0,9/100.000 em 2003). Há de se ressaltar a mudança de classificação usada, da CID-9 para a CID-10, em 1996 com a implícita mudança de classificação da causa básica do óbito, por exemplo, os com epilepsia e infecção pulmonar, tiveram a primazia da classificação da epilepsia na CID-10. Isso poderia colaborar no aparente aumento da taxa de mortalidade por epilepsia. Além disso, os autores ${ }^{4}$ consideraram que a análise epidemiológica da taxa de mortalidade mostrou como no estudo anteriormente mencionado, ${ }^{9}$ predominância masculina e nas faixas etárias avançadas. No entanto, esses autores ${ }^{4}$ sugerem aumento expressivo de mortalidade de epilepsia no Nordeste brasileiro no período estudado. A predominância masculina e dos mais idosos faz supor que muitas das causas do óbito são advindas de epilepsia sintomática aguda, por TCE, AVE, infeç̧ões do SNC, por exemplo, do que as relacionadas primariamente à epilepsia.

\section{Os principais fatores de risco para morte prematura em pessoas com epilepsia}

Idade e gênero - a taxa de mortalidade padronizada (TMP) mais alta para crianças com epilepsia reflete a baixa mortalidade na população de referência pelo menos em países desenvolvidos. ${ }^{1}$ As TMP diminuem com idade crescente, mas, o excesso mais significativo de mortes é notável em faixas etárias mais velhas. A maioria dos estudos informou uma maior TMP para homens (TMP 2,0-3,6) com epilepsia comparada com a de mulheres (TMP 0.8-2.3). O National General Practice Study of Epilepsy (NGPSE) registrou porém TMP de 2,7 para os homens e 3,4 para mulheres. ${ }^{1}$ Essa predominância masculina e de faixas etárias mais velhas também foi constatada nos estudos já comentados a partir de declarações de óbitos.., 9 
Tipo de CE e síndrome de epilepsia - estudos populacionais sugeriram que epilepsias idiopáticas sejam associadas a um aumento mínimo de TMP entre 1,1 e 1,9, mas, apenas o estudo de Rochester informou um aumento definido nestas síndromes. Porém, epilepsia sintomática teve TMP constantemente aumentado entre 2,2 e 6,5, segundo Hitiris et al. ${ }^{1}$ Nicoletti et al. ${ }^{6}$ avaliaram a história natural e mortalidade da epilepsia crônica em uma coorte de prevalência não tratada (seguimento de 10 anos) de pessoas com epilepsia em uma área rural de Bolívia. A taxa de mortalidade foi de 10/1.000 pessoas-ano em risco, sem um aumento de risco significativo em relação à população geral. Houve um risco aumentado significativo para pacientes com epilepsia sintomática remota (TMP 3,0; IC 95\%: 1,2-6,3), mas não com epilepsia idiopática. Três dos 10 sujeitos morreram de causas possivelmente relacionadas à epilepsia. Hesdorffer et al. ${ }^{5}$ estudaram o risco de mortalidade em uma população (Rochester) com CE sintomáticas agudas (devido à infecção do SNC, AVC e TCE) e primeira $\mathrm{CE}$ não provocada devido às lesões estáticas cerebrais. Eles concluíram que os indivíduos com uma primeira CE sintomática aguda eram 8,9 vezes mais sujeitos a morrer em 30 dias em comparação aos com primeira CE não provocada, depois de ajuste para idade, gênero e EME. Nos sobreviventes, o risco de mortalidade em 10 anos nos com $\mathrm{CE}$ não provocada foi significativamente maior do que nos com aguda sintomática, e nestes a probabilidade de ter $\mathrm{CE}$ recorrente não provocada foi $80 \%$ menor. Os autores ${ }^{5}$ concluem que essas diferenças não favorecem a inclusão de $\mathrm{CE}$ agudas sintomáticas como epilepsia.

Déficits neurológicos congênitos e déficit de aprendizagem - algumas das taxas de mortalidade mais altas foram informadas em pessoas com epilepsia sintomática associada com déficits neurológicos congênitos. Mortes eram mais comuns quando as CE eram generalizadas desde o início. Pneumonia foi a causa mais comum de morte, e $\mathrm{CE}$ responderam por pouco, $6.7 \%$. O fator de risco para mortalidade nesses pacientes eram mobilidade pobre, frequência alta de CE e polifarmácia crescente com drogas antiepilépticas (DAE). ${ }^{1}$

Duração e gravidade da epilepsia - a taxa de mortalidade aumentada é muito proeminente nos primeiros anos de CE. A mortalidade excessiva precoce nos estudos de incidência é devida a lesões cerebrais de risco à vida que causam a epilepsia. ${ }^{1}$ Uma baixa frequência de CE parece reduzir risco de mortalidade. ${ }^{1}$ Sillanpaa e Schmidt ${ }^{7}$ estudaram se as $\mathrm{CE}$ agrupadas eram relacionadas à farmacorresistência e mortalidade em epilepsia de início na infância, em um estudo prospectivo populacional. Eles concluíram que as $\mathrm{CE}$ agrupadas em pessoas em tratamento, mas não antes de tratamento, são associados a prognóstico pior da epilepsia e maior mortalidade.

\section{Mortalidade relacionada à epilepsia}

Agora vamos estudar as etiologias relacionadas à epilepsia que segundo Hitiris et al. ${ }^{1}$ cobrem $1-13 \%$ das mortes em estudos populacionais. Hitiris et al. ${ }^{1}$ relacionaram além das clássicas (SUDEP - 0-4\%, CE e EME - 0-10\%, Suicídio - 0-7\%, Acidente - 0-12\%) também cirurgia para epilepsia, estimulação do nervo vago e gravidez.

As taxas mais altas de SUDEP estão relacionadas a pacientes com epilepsia farmacorresistente e, principalmente, aos investigados para ou submetidos à cirurgia para epilepsia (2,2-9,3/1000 pacientes ano). ${ }^{1}$ A SUDEP é conceituada como "A súbita, inesperada, testemunhada ou não, morte não traumática/não por afogamento em um paciente com epilepsia, com ou sem evidência de CE e excluído EME na qual a autópsia não revela uma causa toxicológica ou anatômica para morte", Nashef, 1997, apud Hitiris et al. ${ }^{1}$ Hitiris et al. ${ }^{1}$ informam que quando a SUDEP é suspeitada, mas autópsia plena e dados toxicológicos não existem, podem ser categorizadas mortes como SUDEP provável ou possível. ${ }^{1}$ Segundo Hitiris et al. ${ }^{1}$ e Ryvlin et al.,2 em relação a aspectos ligados à etiopatogenia da SUDEP: uma das suas causas principais parece ser a consequência direta de uma $\mathrm{CE}$ e assim um evento CE-relacionado; há risco aumentado em pacientes com crises noturnas, crises generalizadas tônico-clônicas e aderência pobre à medicação prescrita; geralmente acontece durante o sono; em alguns estudos parece haver predominância masculina, embora outros não acharam uma diferença de gênero; alguns estudos sugeriram que os pacientes com epilepsias sintomáticas tenham maior risco do que os com idiopáticas, embora outros não indicaram nenhuma diferença clara; a apneia central ou obstrutiva pós-ictal parece representar mecanismo provável, além de arritmia cardíaca, transtorno autonômico e hipoxia, no entanto, a causa parece ser multifatorial. Scorza et al. ${ }^{12}$ lembram também das baixas temperaturas do inverno como fator de risco para SUDEP.

Segundo Ryvlin et al. $^{2}$ a Liga Francesa contra a epilepsia definiu a mortalidade ligada à epilepsia, principalmente a SUDEP, como seu eixo prioritário de pesquisa e ação. Vários projetos de pesquisa originais estão em curso, sendo que alguns deram uma extensão européia dentro do plano do MORTality in Epilepsy Monitoring Unit Study - Mortemus. Complementação sobre SUDEP em seus aspectos preventivos é dada na seção sobre profilaxia das mortes evitáveis.

Quanto ao EME, alguns aspectos são ressaltados: continua tendo mortalidade significativa apesar de melhorias percebidas no diagnóstico e tratamento; incidência anual de $10-60$ por 100.000 /população geral e conta entre 0,5-10\% de todas as mortes por epilepsia, com a TMP de 2,8 (IC 95\%: 2.1-3.5); distribuição de idade é bimodal, 
com a maior incidência no primeiro ano de vida e depois da idade de 60; história de epilepsia estava presente em 42-50\% dos pacientes; letalidade maior em adultos relacionada à hipoxia, por parada cardíaca, infecção cerebral e doença cerebrovascular. ${ }^{1}$

Em relação ao suicídio: prevalência na vida de suicídio e tentativa de suicídio entre pacientes com epilepsia foi informado variar de 5-14\%; risco é aumentado também em crianças e adolescentes; pacientes suicidas com epilepsia mais provavelmente pertencem a coortes selecionadas como os de epilepsia farmacorresistente e/ou comorbidades psiquiátricas. ${ }^{1}$ Em particular, interessa-nos por razões preventivas a ideação suicida que pode representar a fase precoce de um futuro dano autoinfligido ou suicídio. Dos 97 pacientes estudados com a participação da autora, ${ }^{10}$ 38 mulheres e 59 homens, com média de idade de 39,73 anos (DP 12,34, 18-66), a prevalência de período de ideação suicida foi de 13,40\% (IC 95\%: 7,87-21,73). As diferenças entre os com ou sem ideação suicida foram estatisticamente significativas em relação à qualidade do sono medida pelo Pittsburgh sleep quality índex $(p=0,003)$, sintomas depressivos e ansiosos, medidos pelos inventários correspondentes de Beck ( $\operatorname{ambos} \mathrm{p}=0,000$ ), mas não à idade, frequência de CE e DAE ou escolaridade. A prevalência de ideação suicida da amostra estudada está na média acima da do estudo de Casey et al. com metodologia similar, apud Gomes et al. (população geral: 2,3\% na Espanha; 7,4\% na Inglaterra; 7,4\% na Noruega; 9,8\% na Finlândia; 14,6\% na Irlanda).

Quanto aos acidentes, as mortes acidentais relacionadas à epilepsia geralmente são devidas a trauma, acidentes de tráfico de estrada, quedas, queimaduras e afogamento. É considerado que as pessoas com epilepsia estão em risco aumentado de acidentes fatais em comparação com a população geral. ${ }^{1}$

A relação da mortalidade e a cirurgia para epilepsia é controversa: se ela reduz a mortalidade das pessoas com epilepsia farmacorresistente. ${ }^{3}$ Sua patofisiologia continua incerta, mas as mortes testemunhadas aconteceram durante ou imediatamente após uma CE. Vários estudos avaliaram o impacto da cirurgia de epilepsia na mortalidade. Quatro séries se concentraram em pacientes operados, comparando os com CE ou sem no pós-operatório. Três desses estudos informaram um risco de SUDEP significativamente mais baixo nos pacientes curados pela cirurgia em relação aos demais. Quatro outras séries compararam os pacientes operados em relação aos tratados clinicamente com epilepsia refratária focal, sendo que três destes estudos não mostraram qualquer diferença significativa na taxa de morte ou SUDEP. Segundo Ryvlin e Montavont, ${ }^{3}$ todas séries sofreram limitações metodológicas o que impossibilita qualquer conclusão definitiva relativa ao impacto da cirurgia de epilepsia na mortalidade.
Outro aspecto sobre intervenção em pessoas com epilepsia é o da estimulação do nervo vago. Aparentemente, a mortalidade global e por SUDEP nesses pacientes foi similar às referidas em ensaios clínicos sobre novas DAE e coortes de pacientes com epilepsia grave. ${ }^{1}$

Um aspecto particular de uma população é a das mulheres grávidas com epilepsia. Vários investigadores não têm achado um aumento significativo no seu risco de mortalidade. Foram implicados nos poucos casos observados, cuidado de epilepsia de baixo padrão e frequência pobre ao hospital como possíveis fatores potenciais dessa mortalidade. ${ }^{1}$

\section{Mortalidade não relacionada à epilepsia}

Vamos agora abordar a mortalidade não relacionada à epilepsia (\% em estudos populacionais). As principais causas segundo Hitiris et al., ${ }^{1}$ são devidas às neoplasias (18-40\%), doenças cerebrovasculares (12-17\%), doença isquêmica coronariana (12-37\%) e infecções respiratórias (8-18\%). Mais especificamente em relação aos neoplasmas, Hitiris et al. ${ }^{1}$ relatam que há um aumento da TMP para as neoplasias, mesmo após exclusão de tumores cerebrais primários. De acordo com Singh et al., ${ }^{8}$ o excesso modesto de todas as mortes por câncer e excessos grandes de mortes devido a cânceres em certos locais na epilepsia grave (TMP 1,42; IC 95\%: 1,18-1,69) e a falta disso na epilepsia presumidamente mais moderada (TMP 0,93; IC 95\%: 0,84-1,03) sugerem dessemelhanças no perfil dos fatores de risco das duas populações: estilo de vida ou DAE? Os neoplasmas relacionados ao tempo de diagnóstico da epilepsia é bem maior, com menos de 5 anos de diagnóstico, no cérebro. ${ }^{8}$ Em relação aos tipos de cânceres, os autores reportam: a TMP para todos os cânceres foi significativamente mais elevada nos casos graves (1,42; IC 95\%: 1,18-1,69), mas não nos casos mais moderados (0,93; IC 95\%: 0,84-1.03). A TMP para neoplasias do SNC foi significativamente elevada nos casos mais brandos, sendo a maioria das mortes por câncer em idades mais jovens ( $<45$ anos). Nos casos graves, os cânceres de esôfago, fígado e vesícula biliar e o linfoma não Hodgkin foram elevados.

\section{Profilaxia das mortes evitáveis}

Agora vamos abordar a prevenção da SUDEP e outras mortes epilepsia relacionadas. O Epilepsy Bereaved, ${ }^{11}$ fundado em 1993, é uma organização voluntária de origem no Reino Unido que trabalha para prevenir mortes desnecessárias por SUDEP. Segundo o seu site, ela é a primeira organização voluntária que conduziu uma auditoria clínica nacional sobre mortalidade em epilépti$\cos$ (2002). O objetivo principal da auditoria foi a de evitar mortes prematuras potencialmente evitáveis. A conclusão foi a de que a investigação de mortes epilepsia-relacionada 
era pobre, o que favoreceu aprender com estas mortes. A auditoria já conduziu à orientação específica sobre SUDEP incluída no NICE Epilepsy guidelines 2004, ${ }^{14}$ um plano de ação de Governo sobre epilepsia na Inglaterra, e, também, no Royal College of Pathologists Guidelines on Autopsy Practice Deaths associated with Epilepsy. Em relação às diretrizes clínicas sobre epilepsia no adulto e na criança do NICE há uma seção sobre "Informação que deveria ser provida se uma pessoa teve uma possível CE": como se reconhecer outra CE e o que fazer se ela acontecer (inclusive informação de pronto socorro). Isso teria que ser dado aos pacientes e a sua família ou cuidadores. No entanto, Morton et al. ${ }^{13}$ fizeram uma pesquisa sobre a prática clínica real entre neurologistas do Reino Unido, a partir de questionário enviado a todos os especialistas listados na Association of British Neurologists database, com a indagação em que circunstâncias eles falaram para os pacientes sobre SUDEP. Dos respondentes validados, 5\% discutiram SUDEP com todos os pacientes, 26\% com uma maioria, $61 \%$ com alguns, e 7,5\% com nenhum. O mais frequente argumento para a informação era se os pacientes perguntavam sobre isto e se o neurologista aconselhava pessoas com fatores de risco conhecidos para SUDEP. Por conseguinte, Morton et al. ${ }^{13}$ concluíram que a variação de respostas encontradas não necessariamente afinadas com as diretrizes do NICE, reflete a variação na necessidade dos pacientes por conhecimento sobre a sua condição. Ryvlin et al., ${ }^{2}$ ponderam que os profissionais estão cientes de que o risco de SUDEP é muito baixo entre a maioria dos seus pacientes, sendo desnecessário exacerbar o sofrimento psicológico causado pela epilepsia com a informação excessiva. Isso também remete ao "direito de não saber". Ryvlin et al., ${ }^{2}$ lembram que esse é um conceito do humanismo médico, tendo em conta a ambivalência do paciente em relação às informações que podem ser emitidas, a decodificação real que podem fazer e as consequências potencialmente deletérias, incluindo à saúde, da informação percebida como alarmante e sem recurso. Ryvlin et al.,2 também lembram que estudos mostram que a maioria dos pacientes que sofrem de câncer incurável, não querem enfrentar os elementos específicos que determinam a sua vida ou evolução negativa de sua doença. Mutatis mutantis, é o caso da epilepsia, uma vez que nenhuma medida eficaz de prevenção foi formalmente demonstrada. No entanto, Ryvlin et al., ${ }^{2}$ reforçam que é necessário responder com precisão às perguntas feitas pelos pacientes ou seus familiares sobre o assunto. Posso morrer de uma crise? Sim, com ênfase para os riscos acidentais que podem ser prevenidos (incluindo o afogamento), e especificando a natureza do risco, dependendo do tipo de epilepsia. Assim, nas crianças, o risco de SUDEP é quase zero, exceto para formas muito graves da doença subjacente ou encefalopatia. É a mesma coisa na epilepsia bem controlada em adultos. No entanto, esse risco não pode ser ignorado nas formas farmacorresistentes de adultos jovens, onde a evocação do assunto pode levar a salientar a importância da adesão ao tratamento e começar uma discussão sobre a conveniência de tratamento cirúrgico.

Quanto à redução das mortes evitáveis, o Epilepsy Bereaved $^{11}$ gera recomendações sobre "Como reduzir o risco de SUDEP e outras causas de morte por epilepsia", especialmente com precauções sobre: a redução de crises e quando elas ocorrem, como reduzir o seu risco (à noite, primeiros socorros e medidas de ressuscitação). Scorza et al. ${ }^{12}$ indicam hipoteticamente o exercício físico regular como uma medida preventiva para a SUDEP. Vide Quadro 1.

Quadro 1. Sugestões para reduzir risco de mortes evitáveis em epilepsia (adaptado de Epilepsy Bereaved, ${ }^{11}$ Gomes et al., ${ }^{10}$ Scorza et al. ${ }^{12}$ ).

\begin{tabular}{|c|c|}
\hline & Medidas \\
\hline Bases & $\begin{array}{l}\text { - Proceder a pronto diagnóstico e tratamento por } \\
\text { especialista com interesse em epilepsia. } \\
\text { - Evitar fatores precipitantes para crises epilépticas } \\
\text { quando possível. } \\
\text { - Ter boa aderência à prescrição. } \\
\text { - Considerar alternativas para reduzir CE como } \\
\text { cirurgia para epilepsia e encaminhamento para centro } \\
\text { especializado de epilepsia. } \\
\text { - Dar informações ao paciente para que ele melhore a } \\
\text { compreensão de sua doença. } \\
\text { - Revisar diagnóstico e discutir sobre outras opções de } \\
\text { tratamento. } \\
\text { - Fazer consultas regulares. } \\
\text { - Cuidar da saúde mental. } \\
\text { - Ter atividade física regular. }\end{array}$ \\
\hline Segurança & $\begin{array}{l}\text { - Preferir banhos de ducha. } \\
\text { - Evitar fechar portas com chaves. } \\
\text { - Usar alarmes de incêndio/fumaça e preferir fogão de } \\
\text { microonda. } \\
\text { - Evitar situações de risco: alturas, beira d'água ou } \\
\text { nadar sozinho. }\end{array}$ \\
\hline À noite & $\begin{array}{l}\text { - Dormir ao nível do chão. } \\
\text { - Ter supervisão noturna }\end{array}$ \\
\hline
\end{tabular}

\section{Conclusões}

Conclui-se que os pacientes com epilepsia tem a mortalidade aumentada em comparação à população geral, principalmente nos anos iniciais da doença devido à causa subjacente das epilepsias sintomáticas agudas, nos com epilepsia farmacorresistente e sintomática remota. Em relação ao EME, ele é frequentemente associado à sequela de paradas cardíacas, infecção cerebral e alta letalidade. A incidência de EME parece estar aumentando, por envelhecimento da população e pacientes que experimentam EME mioclônico que se segue à parada cardíaca. A questão da mortalidade aumentada ou não no pós-operatório de cirurgia para epilepsia não está respondida, pois as taxas variam segundo diversos estudos, talvez pelas diferenças em seleção dos paciente para a cirurgia. 
Quanto aos suicídios, eles foram comuns em coortes mais selecionadas, particularmente nos com epilepsia refratária e/ou comorbidade psiquiátrica Questão atualmente em voga é o da SUDEP, um fenômeno epilepsia-relacionado, de maior ocorrência em pessoas jovens com epilepsia. São nela implicadas a apneia central e obstrutiva, arritmias cardíacas, crises tônico-clônico generalizadas, frequência alta de crises, déficit de aprendizagem concomitante, polifarmácia com DAE e mudanças frequentes de dosagem. A temida mortalidade excessiva e desnecessária gera em contrapartida a necessidade da sua prevenção. No entanto, ainda não há consenso como a informação sobre mortes evitáveis em epilepsia deveria ser dada a pacientes e familiares, embora deva ser considerada a individualização da informação para cada paciente. Para estimativas precisas de mortalidade em epilepsia, estudos deveriam ser longitudinais, baseados em população de pacientes com epilepsia recentemente diagnosticada.

\section{REFERENCIAS}

1. Hitiris N, Mohanraj R, Norrie J, Brodie MJ. Mortality in epilepsy. Epilepsy Behav. 2007;10(3):363-76.

2. Ryvlin P, Tomson T, Montavont A. Excess mortality and sudden unexpected death in epilepsy. Presse Med. 2009;38(6):905-10.

3. Ryvlin P, Montavont A. Does epilepsy surgery really lower mortality? Neurochirurgie. 2008;54(3):282-6.

4. Ferreira IL, Tabosa e Silva TP. Mortality from epilepsy in Brazil, 19802003. Cien Saude Colet. 2009;14(1):89-94.

5. Hesdorffer DC, Benn EK, Cascino GD, Hauser WA. Is a first acute symptomatic seizure epilepsy? Mortality and risk for recurrent seizure. Epilepsia 2009;50(5):1102-8.
6. Nicoletti A, Sofia V, Vitale G, Bonelli SI, Bejarano V, Bartalesi F, Tran DS, Preux PM, Zappia M, Bartoloni A. Natural history and mortality of chronic epilepsy in an untreated population of rural Bolivia: a follow-up after 10 years. Epilepsia. 2009;50(10): 2199-206.

7. Sillanpää M, Schmidt D. Seizure clustering during drug treatment affects seizure outcome and mortality of childhood-onset epilepsy. Brain. 2008;131(Pt 4):938-44.

8. Singh G, Fletcher O, Bell GS, McLean AE, Sander JW. Cancer mortality amongst people with epilepsy: a study of two cohorts with severe and presumed milder epilepsy. Epilepsy Res. 2009;83 $(2-3): 190-7$

9. Gomes MM. Mortalidade por epilepsia: Brasil (capitais), 2003-2007. J Epilepsy Clin Neurophysiology 2010;16(2 sp 1):74.

10. Gomes MM, Neves GSLM, Venturi M. Ideação suicida e qualidade de sono em pacientes com epilepsia. J Epilepsy Clin Neurophysiology 2010;16(2 sp 1):70.

11. Epilepsy Bereaved. SUDEP safety information. How to reduce risks of SUDEP and other causes of epilepsy death. In: http://www.sudep. org/ (10.04.2010).

12. Scorza FA, Colugnati DB, Pansani AP, Sonoda EY, Arida RM, Cavalheiro EA. Preventing tomorrow's sudden cardiac death in epilepsy today: what should physicians know about this? Clinics (Sao Paulo) 2008;63(3):389-94.

13. Morton B, Richardson A, Duncan S. Sudden unexpected death in epilepsy (SUDEP): don't ask, don't tell? J Neurol Neurosurg Psychiatry 2006;77(2):199-202.

14. National Institute for Clinical Excellence. Diagnosis and care of children and adults with epilepsy. London: NICE, 2004. In: http://www.nice.org.uk/nicemedia/pdf/CG020publicinfoenglish.pdf (10.04.2010).

Endereço para correspondência:

Marleide da Mota Gomes

Instituto de Neurologia da UFRJ - Programa de Epilepsia

Av. Venceslau Braz, 95 - Botafogo

CEP 22290-140, Rio de Janeiro, RJ, Brasil 